\title{
Hierarchy theory as a conceptual framework for scale issues in avalanche forecast modeling
}

\author{
Pascal HÄGELI, ${ }^{1}$ David M. MaCLUNG ${ }^{2}$ \\ ${ }^{1}$ Atmospheric Science Programme and ${ }^{2}$ Department of Geography, University of British Columbia, 1984 West Mall, \\ Vancouver, British Columbia V6 T 122, Canada \\ E-mail: pascal@geog.ubc.ca
}

\begin{abstract}
Although scale issues have been defined to be one of the most crucial topics in geosciences, they have received only limited attention in avalanche research. A thorough understanding of these issues in avalanche forecasting, however, is fundamental for the development of useful prediction models. After the definition of relevant terms, the different aspects of scale issues are introduced in detail. We present hierarchy theory (Ahl and Allen, 1996) as a potential framework for the multi-scale characteristics of the avalanche phenomenon. We suggest a temporal hierarchy, where the main contributing factors are ordered into seven levels according to their temporal-scale characteristics with respect to avalanche forecasting. Within each level there are individual spatial hierarchies, which result in a two-dimensional structure. This process-oriented framework is compared to the data classification scheme of LaChapelle (1980), which is based on informational entropy. Scale issues in prediction modeling and the consequences of the new framework for modeling efforts are discussed in detail.
\end{abstract}

\section{INTRODUGTION}

Avalanches are the result of numerous interactions of various contributing factors and processes that act over a large range of different spatial and temporal scales. Examples range from avalanche initiation on the scale of meters to synoptic weather systems that can span thousands of kilometers. Avalanches are a complex phenomenon in which small changes in individual components can result in considerably different activity characteristics. Predicting the behavior of such a complex system is highly challenging since it requires addressing multiple levels of scale simultaneously. Over the last century, avalanche practitioners, such as mountain guides, have developed highly successful inductive decision-making strategies to deal with this complexity (LaChapelle, 1980). These rules, sometimes applied intuitively, are able to deal comprehensively with the numerous processes and multi-scale nature of the avalanche phenomenon. In recent years, considerable effort has been put into the development of statistical and numerical models to facilitate the work of avalanche professionals. In order to develop appropriate strategies and models, it is necessary to have a thorough understanding of the scale characteristics of the phenomenon and all the contributing factors. Although scale has been called one of the most important issues in current geosciences (University Consortium for Geographic Information Science, http:// www.ucgis.org/research_white/scale.html), scale and scale issues have received only limited attention in avalanche research. The main focus has been on small-scale studies (e.g. Kronholm and others, 2002; Landry and others, 2002), which have produced interesting insights but are of limited value for avalanche forecasting at operational scales, such as entire ski resorts, highway corridors or numerous drainages in the case of back-country operations.
The goal of this paper is to highlight scale issues in avalanche forecasting and present a conceptual framework for dealing with them across a wide range of scales.

\section{SGALE, SGALING AND SGALE ISSUES}

The term "scale" refers to a characteristic length or time of a process, measurement or model (Blöschl and Sivapalan, 1995). Process scales can be described by single parameters in space and time, such as the duration or frequency of a process. The scale characteristics of a measurement or model are defined by scale triplets in space and time: extent, spacing and support (Blöschl and Sivapalan, 1995). In the case of a monitoring network, spacing (also called grain by Ahl and Allen, 1996) refers to the distance between samples; extent stands for the overall coverage of the data; and support represents the integration volume or area of the sample. Knowledge of these scale characteristics is crucial because they determine which components of the true process scale can be captured with an observation. The apparent process scale displayed in measurements is the result of the observational scales superimposed on the true process scale. Similar arguments can be made for model scales and the apparent scale characteristics of their outputs.

"Scaling" involves transfer of information across scales. "Scale linkage" (Phillips, 1999) refers to the information transfer between processes that act over fundamentally different scales, which is crucial for understanding the overall scale characteristics of a complex system. Another aspect is the scaling of information between processes and observations or models, which is described in detail by Blöschl and Sivapalan (1995).

Difficulties that arise when dealing with scale and scaling are generally referred to as "scale issues". Phillips (1999) 
classifies them into four general categories. First is the basic problem of determining the natural spatial and temporal scales of a process. Here, the goal is to gain fundamental understanding of the process or phenomenon. Popular geostatistical methods are semi-variogram or wavelet analyses. Second, there is the problem of matching the observational and model scale with the natural scale of the process in question. This aspect deals with the technicalities of setting up suitable monitoring systems and choosing the appropriate spatial and temporal model resolutions. Third, there are problems with information transfer across scales when the observation and model scales are different from the true process scale. This involves the distribution of localized information through space and time. An example for a geostatistical extrapolation method is kriging. Fourth, there are issues of dimensionality and similarity that address the range of scales over which patterns or relationships are constant or even valid. A related example of this scaling aspect is size effects in fracture mechanics as discussed by Bazant and others (2003).

These four scaling issues are interrelated and they are all significant in avalanche forecasting. The most urgent aspect, however, is a clear understanding of the scale characteristics and scale linkage of the factors and processes that lead to avalanching. This basic knowledge is crucial for the development of appropriate monitoring networks and useful forecasting models.

Scale issues have been much debated in many geosciences, which has resulted in the development of many discipline-specific solutions. Unfortunately, the goals of avalanche forecasting differ fundamentally from those of neighboring disciplines such as geomorphology or hydrology. Avalanche forecasting is the prediction of current or future snow instability in space and time relative to a given triggering level (McClung, 2002a). This goal is different from, for example, snow hydrology, where one of the main interests is the determination of the total water equivalent stored in a given area. This question involves upscaling of point measurements to an entire area. The focus in avalanche forecasting is on determining possible locations of trigger spots within an area. This, in turn, requires detailed point-to-point extrapolation and interpretation of individual observations. The ability to extrapolate is highly dependent on the similarity of locations and our knowledge of the influencing parameters. This fundamental difference prevents the appliation of existing approaches for scale issues, and it is necessary to develop new strategies specifically for avalanche forecasting.

\section{APPLICATION OF HIERARGHY THEORY TO AVA- LANGHE FOREGAST MODELING}

Hierarchy theory (Ahl and Allen, 1996) has been suggested as a conceptual framework for organizing complex systems and highlighting related scale issues. Examples of its application are de Boer (1992) in geomorphology and Allen and Hoekstra (1992) in ecology.

The theory is less practical for determining natural scales, which is generally the field of geostatistics (e.g. Webster and Oliver, 2000). Because of "targeted sampling" techniques (McClung, 2002b) and generally incomplete avalanche observations (Hägeli and McClung, 2003), it is impossible to apply these methods to avalanche datasets that match our scale of interest. Our discussion will therefore be limited to a theoretical framework that highlights scale issues relevant to avalanche forecasting and related modeling efforts.

Hierarchy theory is a holistic approach that includes the observer in the problem space. Although generally the observer does not control a system, the observed behavior is viewed in the context of the question posed and the observer's observational protocol. The incorporation of the observer in the problem space seems particularly appropriate in avalanche forecasting because of the crucial role of human perception (McClung, 2002a).

\section{Temporal hierarchy}

The theory uses a relatively simple set of rules to organize the processes and interactions in a complex system. Contributing facors are grouped into hierarchical levels according to their temporal- or spatial-scale characteristics. In the time domain, higher levels exhibit lower-frequency behavior and represent the context or constraint for entities at lower levels. With regard to lower-level activity, higher-level behavior can often be regarded as constant and may contain the memory component of the complex system.

In the case of avalanche forecasting, one can distinguish seven different interacting temporal levels. These are terrain, ground cover, snowpack, weather, artificial triggers, the avalanche phenomenon (our level of interest) and snow physics. These levels can be ordered into a hierarchy according to their typical time-scales with regard to a typical operational forecasting period of 1 day (Fig. 1). Evolving in geological time-scales, terrain clearly changes most slowly and represents the ultimate constraint for the occurrence of avalanches. It can be regarded as constant for normal avaanche-forecasting applications. Ground-cover entities, such as glaciation or vegetation, are important parameters for avalanche forecasting, particularly during the early season and for full-depth avalanches. Typical temporal scales of this level are shorter than geological time-scales, but still longer than regular forecasting periods. The characteristics of the existing snowpack including the behavior of persistent weak layers (Jamieson, 1995) are tremendously important for predicting avalanches. Hägeli and McClung (2003) show that the typical time-scale for avalanche activity on such weak layers ranges from approximately 3 weeks to the entire season. Weather represents the next lowest level. Typical synoptic weather systems have a life span of a few days. When forecasting avalanches with regard to nonnatural triggers, artificial triggers represent an additional hierarchical level. The shorter temporal characteristics of the triggers place this level right above our level of interest. Situated below the avalanche level is the level of snow physics, which contains the principles of fracture mechanics with typical temporal scales of seconds.

While levels above the avalanche level represent environmental conditions that allow avalanches to occur or not, the level below contains the underlying principles necessary for avalanches to occur. This leads to the concept of the "constraint envelope" (O’Neill and others, 1989), which states that avalanches must occur within a window that is defined by a combination of the low-level principles and upper-level environmental constraints. An excellent example of this concept is the fact that the majority of slab avalanches occur on slope angles of $25-55^{\circ}$ (McClung and 
SPATIAL HIERARCHIES

within each level/sphere

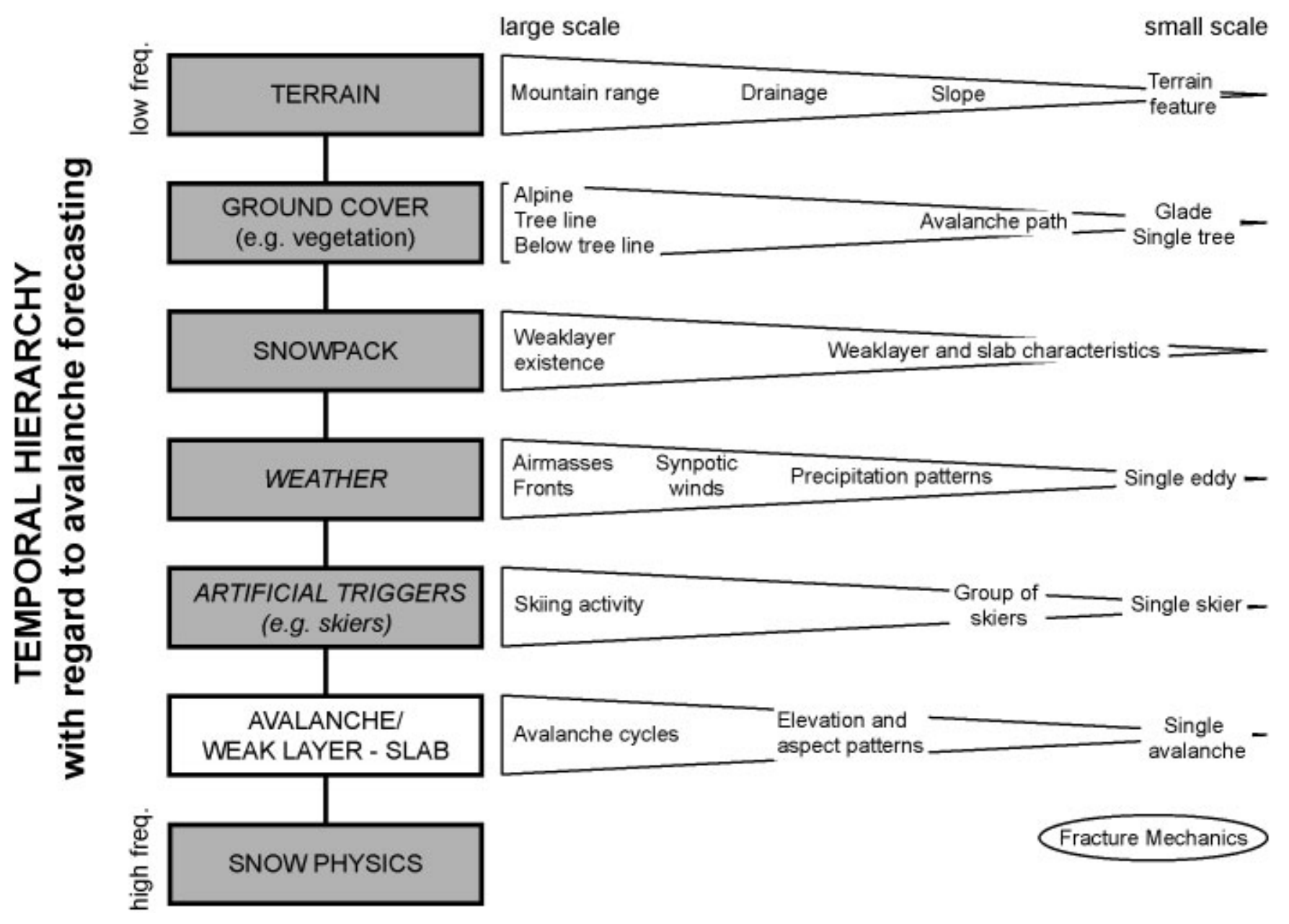

Fig. 1. Two-dimensional hierarchical framework for avalanche forecast modeling with examples.

Schaerer, 1993). The principles of fracture mechanics generally do not allow avalanches on slopes below $25^{\circ}$, while frequent sluffing (loose snow avalanches) on slopes above $55^{\circ}$ prevents slabs from forming (McClung and Schaerer, 1993). More important from an operational point of view is that this constraint envelope changes over time. During periods of low stability, the envelope is large and numerous combinations of environmental factors lead to widepread avalanching. During periods of high stability, the envelope is small and avalanches are rare. A useful forecast model should point out all possible combinations that might lead to avalanching under the current conditions.

In order to forecast avalanches accurately, it is necessary to include information from all the levels presented. Depending on the time-frame of the forecast, more or fewer levels have to be incorporated in a dynamic way. When forecasting over time-spans of minutes during control work, all levels except artificial triggers are static, while in the case of forecasting over a few days, the weather and snowpack levels must also be incorporated dynamically.

The present hierarchy is a partially nested one, where the higher snowpack level contains the avalanche level. For most forecasting applications, there is only a downward transfer of information/matter/energy in this hierarchy. Only when forecasting on individual slopes is there a significant feedback of an avalanche event on the characteristics of the local snowpack. In this case, the snowpack can be viewed as the memory of the system. There is no effect of avalanches on the levels of weather and artificial triggers. These can be regarded as incorporated disturbances (Ahl and Allen, 1996) that interact only in a one-way fashion with the nested avalanche system.

However, weather also plays a crucial role in the development of the snowpack. The hierarchy illustrated in Figure 1 was specifically designed with respect to operational avalanche forecasting. A hierarchical system for the development of the snowpack will be significantly different from the framework presented, although the systems are closely related. Similarly, a hierarchy that focuses on avalanche prediction for the purpose of hazard mapping might include a different set and a different order of levels. These different hierarchies do not have to be contradictory. Instead, they demonstrate the capacity of hierarchy theory to provide problem-specific frameworks.

\section{Spatial hierarchies}

So far we have ordered the seven levels roughly according to their temporal-scale characteristics. Each of these levels contains processes that exhibit a wide range of spatial scales. These can also be organized in individual hierarchies. As with the temporal hierarchies, large-scale phenomena and processes represent the context for small-scale processes. In order to understand the resulting spatial-scale 
characteristics of the avalanche phenomenon, it is necesary to have knowledge of the spatial hierarchies within each level of the temporal hierarchy (Fig. 1). It is beyond the scope of this paper to discuss the scale characteristics of each of these levels in detail. We will therefore limit our analysis to a few crucial aspects.

Terrain has been described as fractal or self-similar (see, e.g., Klinkenberg and Goodchild, 1992), which implies that there exists a continual hierarchy of topographic features from large to small sizes. Numerous ecological studies have examined the scale aspects of vegetation. Allen and Hoekstra (1992), for example, argue that the spatial-scale characteristics of vegetation can be conceptualized with spatially nested hierarchies.

There have been many studies of the spatial variability of the snowpack in the context of snow hydrology (e.g. Elder, 1995; Blöschl, 1999). Spatial studies with regard to avalanche prediction, however, are rare. Hägeli and McClung (2003) examined the spatial characteristics of persistent weak layers across a mountain range based on avalanche observations. They show that persistent weak layers with considerable avalanche activity generally cover significant fractions of a mountain range. Within these general areas of activity, they also show embedded smaller-scale variabilities. For example, surface hoar weaknesses on northern aspects are often found in combination with weak sun-crust interfaces on southerly aspects. Birkeland (2001) found similar characteristics when analyzing stability patterns across a small mountain range. Studies that examined snowpack characteristics on individual slopes (e.g. Birkeland and others, 1995; Jamieson, 1995) reveal numerous varibilities on even smaller scales.

Modern texts in atmospheric science (see, e.g., Storch and Zwiers, 1999) suggest that there is a continuous spectrum of atmospheric processes from small to large scales. As with terrain, they can be organized in nested hierarchical systems.

Artificial triggers, such as skiers, on-snow vehicles or explosives, can also be organized in a nested hierarchy. In the case of skiing, for example, we can distinguish between skiing activity in a mountain range in general; the activity of different groups of skiers; and the influence of the track of an individual skier.

All environmental constraints exhibit some sort of spatial hierarchies. They can be continuous, as in the case of terrain, or have more of a stepwise, discontinuous character like artificial triggers. Fracture mechanics, however, acts only over a narrow range of spatial scales. Most models for dry-snow slab avalanche release mention previously existing imperfections on the order of meters (Schweizer, 1999). Depending on conditions, fracture propagation within the weak layer can cover hundreds of meters. The fact that avalanches are based only on a small-scale principle is a major difference to many other complex systems where hierarchy theory has been applied. For the most part, avalanches are independent events that do not influence each other. Large avalanche cycles are accumulations of individual avalanche occurrences rather than a dynamic system of interacting events. This has important consequences for how avalanche activity can be modeled at different scales.

The scale properties exhibited by avalanches are the result of the interacting spatial characteristics of all environmental constraints and underlying principles. In order to understand spatial avalanche patterns, it is important to relate them to the scale characteristics of the contributing factors. Avalanche activity on persistent weak layers, for example, displays spatial characteristics that are a combination of the storm pattern at the time and the distribution of the active weak layer in the snowpack in relation to terrain, ground cover and existing triggers.

The two-dimensional hierarchy suggested here is comparable to other hierarchical frameworks suggested for other complex sysems. Examples are the layered-cake model for a unified ecology by Allen and Hoekstra (1992) or the two-dimensional hierarchy suggested by Kljin and Udo de Haes (1994) for land classification.

\section{DISGUSSION}

After presenting the basic characteristics of the hierarchical framework, we will compare this framework to an existing data classification scheme and discuss its importance for avalanche forecast modeling.

\section{Comparison to informational entropy data classification}

LaChapelle (1980) classified information used in avalanche forecasting into three categories: (3) meteorological factors; (2) snowpack factors; and (1) stability factors. The data classes are ordered according to informational entropy. This is defined as the relevance and ease of interpretation with respect to estimating instability and human perception (McClung, 2002b).

The most obvious difference between the two frameworks is the order of data classes. While LaChapelle's classification is based on information characteristics and perception, the hierarchical structure presented is processoriented and orders levels according to behavior frequency. LaChapelle (1980) focuses on the evaluation of avalanche conditions at the time, and views weather mainly as an input for the development of the snowpack. The hierarchical framework has a different perspective and sees weather as a trigger agent that acts on the current snowpack. The two perspectives clearly emphasize the two-sided role of the weather level. Besides its short-term effect on instability, weather is also the main driving force for the development of the snowpack and has longer-term consequences for instability.

As the spatial forecasting scale decreases, the difficulty of the forecasting problem and the need for accuracy increases (McGlung, 2000). This problem can be overcome by including more low-entropy data (McClung, 2002b). We believe that the implied link between lower-entropy data and smaller scale is mainly a result of the perception of the different data classes and general observation practices. Meteorological data from study plots are generally used to monitor large-scale weather patterns, while snow-pit analyses are used to examine smaller-scale snowpack characteristics. Avalanche observations and stability tests reveal information about local instability. However, meteorological observations also contain detailed information about the local conditions that act on the snowpack at a study plot. Similarly, there are observations from snow-pit analyses and stability tests that are representative over larger scales. Johnson and Birkeland (2002), for example, suggest that shear quality is easier to correlate to widespread signs of 
instability than the score of individual stability tests. In order to predict avalanches at a specific scale, it is necessary to take information from all levels into account. The challenge lies in finding the variable that properly represents the processes of a level at the scale of interest. While it is sufficient to know about the existence of an active weak layer when forecasting across an entire mountain range, it is necessary to have more detailed information about its characteristics when predicting for individual drainages.

\section{Avalanche forecast modeling}

In operational helicopter skiing, guides forecast avalanches on a range of spatial scales. During morning meetings, general avalanche conditions for the entire operation are discussed on the basis of the conditions observed on the previous day and the overnight changes. During the day, as more small-scale information becomes available, the forecast is continuously updated and becomes more detailed until the focus is on individual terrain features during skiing. Generally, the entire spectrum of contributing factors is taken into account at all stages. Depending on the scale in question and the conditions at hand, however, the importance of individual factors can change. We believe that in order to be successful, computer models must mimic the evolutionary and comprehensive character of avalanche forecasting in time and space.

While human forecasters and guides have developed skills for dealing with scale issues in the forecasting process, these problems have to be explicitly addressed in numerical models. In the following paragraphs we will briefly elaborate on the scale issues in two different modeling approaches.

Statistical forecasting models are based on the idea that similar weather conditions lead to comparable avalanche hazard. Examples are the nearest-neighbor approach (Buser and others, 1987) or the method based on discriminant analysis by McClung and Tweedy (1994). Observations from a representative weather plot are used to monitor largescale weather patterns. In this context, "representative" means that it is possible to draw conclusions about the conditions in the entire operation from this single location. These weather observations are combined with avalanche occurrence data. Depending on the perspective, these observations represent an intermediate scale when looking at the avalanche activity in the entire operation, or stand for a small scale when examining individual avalanche paths. Even though there is a difference in scale between the two data groups, their combination in the model resolves the related scale issues. Since the recorded avalanches are the result of the interaction of all levels, the historic avalanche obervations indirectly contain small-scale information about the static hierarchical levels such as terrain and ground cover as well as trigger information. The fact that these models include only limited information about snowpack characteristics makes them most suited for applications where new-snow instabilities are the main concern. The proximity of the weather level to the avalanche level indicates why these models predict avalanche days with reasonable accuracy.

Another approach is the modeling of snowpack characteristics on the basis of weather observations. In the case of the Swiss SNOWPACK model (Lehning and others, 1999), high-quality, small-scale weather observations are used to simulate the evolution of the snowpack at particular locations. The French SAFRAN-Crocus-MÉPRA model chain (Durand and others, 1999) simulates and analyses the development of the snowpack on a larger scale, using more generalized meteorological observations. In order to use these models for operational avalanche forecasting, it is necessary to explicitly address the question of how these simulations relate to the surrounding area. Since this model approach does not include avalanche observations that indirectly contain this information, it is necessary to develop methods that deal with these issues. These methods need to incorporate information from all hierarchy levels at the appropriate avalanche forecast scale. By simulating the snowpack evolution for different elevation ranges and aspects, the French model chain addresses this issue to a certain extent.

\section{SUMMARY}

The goal of avalanche forecasting is to minimize uncertainty about instability introduced by three principal sources of uncertainty: (1) the temporal and spatial variability of the snow cover, including terrain influences; (2) incremental changes from snow and weather conditions; and (3) human factors including variations in human perception and estimation (McClung, 2002a). The purpose of avalanche-forecasting models is to facilitate this process. The advantage of models is their ability to give objective evaluaions independent of human perception. However, it is tremendously challenging to incorporate all the necessary components into a model to be able to produce forecasts that are truly helpful to avalanche professionals. While human forecasters have developed skills to transfer relevant information across scales, these relationships must be integrated explicitly into a numerical forecasting model. In order to develop useful prediction models, an in-depth understanding of the scale issue related to avalanche forecasting is crucial.

The focus of this paper is on the presentation of hierarchy theory as a conceptual framework for gaining insights into the scale characteristics of the avalanche phenomenon and related scale isues. We suggest a two-dimensional scheme with a temporal hierarchy of seven levels with spatially nested hierarchies. A short discussion of scale characteristics of the individual levels suggests that the resulting avalanche activity can exhibit spatial variabilities on a wide range of scales. In addition to these spatial patterns, the avaanche phenomenon has a probabilistic component which adds another level of complexity. Intensive field research is necessary to improve our understanding of the overall complexity of the avalanche phenomenon. The challenge is to plan large-scale campaigns that allow the study of patterns relevant to forecast applications. A better understanding of the underlying processes will be useful for designing improved monitoring networks and more useful prediction models.

The framework was compared with the data classification of LaChapelle (1980). The main difference between the two points of view is the reversed order of the weather and snowpack level. While LaChapelle sees weather as a "snowpack engineer", it is viewed as a trigger agent in the hierarchical framework. LaChapelle's approach is highly suitable for evaluating the avalanche conditions at hand, 
where it is most effective for examining direct signs of instability. However, for true prediction purposes it seems more useful to view weather as a trigger agent.

In order to develop a useful model at a specific scale, it is necessary to include information from all levels. The challenge is to find the appropriate variable that properly represents the level at the scale of interest. The current perception of data relevant to avalanche forecasting is reflected in the three data classes by LaChapelle (1980). In order to use the available observations most effectively and improve predictability, it may be necessary to review some of the traditional observation protocols.

We discuss some of the scale issues in avalanche forecast modeling. While statistical models contain information about scaling relationships, scale issues must be dealt with explicitly when using numerical snowpack simulations. If we want to apply these models successfully in avalanche forecasting, these questions must be thoroughly addressed in the near future.

\section{ACKNOWLEDGEMENTS}

We thank Canadian Mountain Holidays for their generous support of this research. In particular, we acknowledge $\mathrm{R}$. Atkins, J. Bergstrom and C. Bezzola for their help, and the Gothics operation team for their hospitality. We also express our thanks to K. Birkeland from Montana State University in Bozeman, MT, U.S.A., and C. Landry from the Center for Snow and Avalanche Studies in Silverton, CO, U.S.A., for very fruitful discussions during different stages of this research. We highly appreciate the comments of $\mathrm{H}$. Gruber and an anonymous reviewer, which improved the manuscript considerably. We are grateful for the financial support of Canadian Mountain Holidays, the Natural Sciences and Engineering Research Council of Canada, and the Vice President of Research of the University of British Columbia. P. Hägeli is supported by a University Graduate Fellowship of the University of British Columbia.

\section{REFERENCES}

Ahl, V. and T. F. H. Allen. 1996. Hierarchy theory: a vision, vocabulary, and epistemology. New York, Columbia University Press.

Allen, T. F. H. and T.W. Hoekstra. 1992. Toward a unified ecology. New York, Columbia University Press.

Bazant, Z. P., G. Zi and D. M. McClung. 2003. Size effect law and fracture mechanics of the triggering of dry slab snow avalanches. 7. Geophys. Res., 108(B2), 2119-2229. (10.1029/2002JB001884.)

Birkeland, K.W. 2001. Spatial patterns of snow stability throughout a small mountain range 7 . Glaciol., 47 (157), 176-186.

Birkeland, K. W., K. J. Hansen and R. L. Brown. 1995. The spatial variability of snow resistance on potential avalanche slopes. F. Glaciol., 41 (137), 183-190.

Blöschl, G. 1999. Scaling issues in snow hydrology. Hydrol. Processes, 13(14-15), 2149-2175.

Blöschl, G. and M. Sivapalan. 1995. Scale issues in hydrological modeling — a review. Hydrol. Processes, 9(3-4), 251-290.
Buser, O., M. Bütler and W. Good. 1987. Avalanche forecast by the nearest neighbour method. International Association of Hydrological Sciences Publication 162 (Symposium at Davos 1986 - Avalanche Formation, Movement and Effects), 557-569.

De Boer, D. H. 1992. Hierarchies and spatial scale in process geomorphology: a review. Geomorphology, 4(5), 303-318.

Durand, Y., G. Giraud, E. Brun, L. Mérindol and E. Martin. 1999. A computer-based system simulating snowpack structures as a tool for regional avalanche forecasting. 7. Glaciol., 45(151), 469-484. (Erratum: 46(152), 2000, p. 173.)

Elder, K.J. 1995. Snow distribution in Alpine watersheds. (Ph.D. thesis, University of California, Santa Barbara.)

Hägeli, P. and D. M. McClung. 2003. Avalanche characteristics of a transitional snow climate - Columbia Mountains, British Columbia, Canada. Cold Reg. Sci. Technol., 37(3), 255-276.

Jamieson, J. B. 1995. Avalanche prediction for persistent snow slabs. (Ph.D. thesis, University of Calgary.)

Johnson, R. F. and K. W. Birkeland. 2002. Integrating shear quality into stability test results. In Stevens, J. R., ed. International Snow Science Workshop 2002, 29 September-4 October 2002, Pentiction, British Columbia. Proceedings. Victoria, B.C., B.C. Ministry of Transportation. Snow Avalanche Programs, 508-513.

Klinkenberg, B. and M. F. Goodchild. 1992. The fractal properties of topography - a comparison of methods. Earth Surf. Processes Landforms, $17(3), 217-234$.

Kljin, F. and H. A. Udo de Haes. 1994. A hierarchical approach to ecosystems and its implication for ecological land classification. Landscape Ecol., 9(2), 89-104.

Kronholm, K., J. Schweizer and M. Schneebeli. 2002. Spatial variability of snow stability on small slopes. In Stevens, J. R., ed. International Snow Science Workshop 2002, 29 September-4 October 2002, Pentiction, British Columbia. Proceedings. Victoria, B.C., B.C. Ministry of Transportation. Snow Avalanche Programs, 549-554.

LaChapelle, E. R. 1980. The fundamental processes in conventional avalanche forecasting. f. Glaciol., 26(94), 75-84.

Landry, C. C., K. W. Birkeland, K. Hansen, J. Borkowski, R. L. Brown and R. Aspinall. 2002. Snow stability on uniform slopes: implications for avalanche forecasting. In Stevens, J.R., ed. International Snow Science Workshop 2002, 29 September-4 October 2002, Pentiction, British Columbia. Proceedings. Victoria, B.C., B.C. Ministry of Transportation. Snow Avalanche Programs, 532-539.

Lehning, M., P. Bartelt, B. Brown, T. Russi, U. Stöckli and M. Zimmerli. 1999. SNOWPACK model calculations for avalanche warning based upon a new network of weather and snow stations. Cold Reg. Sci. Technol., 30(1-3), 145-157.

McClung, D. M. 2000. Predictions in avalanche forecasting. Ann. Glaciol., 31, 377-381.

McClung, D. M. 2002a. The elements of applied avalanche forecasting Part 1: The human issues. Nat. Hazards, 26(2), 111-129.

McClung, D. M. 2002b. The elements of applied avalanche forecasting Part II: The physical issues and the rules of applied avalanche forecasting. Nat. Hazards, 26(2), 131-146.

McClung, D. M. and P. A. Schaerer. 1993. The avalanche handbook. Seattle, WA, The Mountaineers.

McClung, D. M. and J. Tweedy. 1994. Numerical avalanche prediction: Kootenay Pass, British Columbia, Canada. f. Glaciol., 40(135), 350-358.

O’Neill, R.V., A. R. Johnson and A.W. King. 1989. A hierarchical framework for the analysis of scale. Landscape Ecol., 3(2-4), 193-206.

Phillips, J. D. 1999. Earth surface systems. Malden, MA, Blackwell Publishers.

Schweizer, J. 1999. Review of dry snow slab avalanche release. Cold Reg. Sci. Technol., 30(1-3), 43-57.

Von Storch, H. and F.W. Zwiers. 1999. Statistical analysis in climate research. Cambridge, etc., Cambridge University Press.

Webster, R. and M. A. Oliver. 2000. Geostatistics for environmental scientists: statistics in practice. Chichester, John Wiley and Sons. 